\title{
The neuropsychology of obsessive-compulsive personality disorder: A new analysis.
}

Fineberg Naomi $\mathrm{A}^{1,2}$. Day Grace $\mathrm{A}^{1,3}$, de Koenigswarter Nica ${ }^{3}$, Reghunandanan Samar ${ }^{1}$, Kolli Sangeetha ${ }^{1}$, Jefferies-Sewell Kiri ${ }^{1,3}$, Hranov $\mathrm{G}^{1,4}$, Laws Keith $\mathrm{R}^{1,3}$.

1. National Obsessive Compulsive Disorders Specialist Service, Hertfordshire Partnership University NHS Foundation Trust, Queen Elizabeth II Hospital, Welwyn Garden City, AL7 4HQ

2. University of Hertfordshire, Postgraduate Medical School, College Lane, Hatfield, Hertfordshire, AL10 9AB

3. University of Hertfordshire, School of Life \& Medical Sciences, College Lane, Hatfield, Hertfordshire, AL10 9AB

4. University Hospital of Active Treatment in Neurology and Psychiatry "St. Naum", Sofia, Bulgaria

Naomi A Fineberg has a MBBS from University of London. Naomi A Fineberg is Visiting Professor in the Highly Specialised Obsessive Compulsive Disorders Service at the Hertfordshire Partnership University NHS Foundation Trust and the University of Hertfordshire, UK.

Grace A Day has a Degree from University of East Anglia. Grace A Day is postgraduate student in the Department of Psychology at the University of Hertfordshire in Hatfield, Hertfordshire.

Nica de Koenigswarter has a MSc in Cognitive Neruopsychology from University of Hertfordshire. Nica de Koenigswarter is postgraduate student in the Department of Psychology at the University of Hertfordshire in Hatfield, Hertfordshire.

Samar Reghunandanan has a [DEGREE] from [UNIVERSITY]. [NAME] is [TITLE] in the [DEPARTMENT] at the [INSTITUTION] in [CITY, STATE]. 
Sangeetha Kolli has a [DEGREE] from [UNIVERSITY]. [NAME] is [TITLE] in the [DEPARTMENT] at the [INSTITUTION] in [CITY, STATE].

Kiri Jefferies-Sewell has an MSc from the University of Hertfordshire. Kiri Jefferies-Sewell is a Research Governance Officer in the Research and Development Department at Hertfordshire Partnership University NHS Foundation Trust, UK and a PhD student at the University of Hertfordshire, Hatfield, UK.

G Hranov has a [DEGREE] from [UNIVERSITY]. [NAME] is [TITLE] in the [DEPARTMENT] at the [INSTITUTION] in [CITY, STATE].

Keith R Laws has a PhD from University of Cambridge. Keith Laws is a Professor of Cognitive Neuropsychology in the Department of Psychology at the University of Hertfordshire in Hatfield, Hertfordshire.

Word Count: 4,383

Figures: 3

Original: 3

Adaptation: 0

Tables: 1

Original: 1

Adaptation: 0

Contact Information: Naomi A. Fineberg, Prof

Dept of Psychiatry, Hertfordshire Partnership NHS Foundation Trust QEII Hospital, Howlands, Welwyn Garden City, AL7 4HQ. Work Phone: 01707365085. Email: naomi.fineberg@hpft.nhs.uk 


\section{Disclosure}

NAF has consulted for Lundbeck, Glaxo-Smith Kline, Transcept, Novartis and Servier; received research support from Novartis, Lundbeck, Glaxo-SmithKline, Wellcome, Cephalon, ECNP, Servier, UK MRC, UK NIHR; received honoraria for lectures at scientific meetings from Janssen, Lundbeck, Servier, Astra Zeneca, Wyeth, Bristol Myers Squibb; received financial support to attend scientific meetings from Lundbeck, Servier, Bristol Myers Squibb, International College of OC Spectrum Disorders, International Society for Addiction, ECNP, BAP, WHO.

\section{Acknowledgements:}

European College of Neuropsychopharamacology, Obsessive Compulsive and Related Disorders Research Network, National Institute for Health Research, Clinical Research Group for Optimising Treatment of Obsessive Compulsive and Related Disorders for providing networking opportunities for this research.

\section{Focus Points:}

- We demonstrated impairment in cognitive flexibility and executive planning in a small group of non-clinical subjects with OCPD

- The neurocognitive deficits show ecological convergence with OCPD traits such as perfectionism, rigidity and slowness.

- These deficits overlap with compulsive disorders such as OCD and are consistent with reclassification of OCPD within a broader Obsessive-Compulsive and Related Disorders grouping. 


\section{Abstract:}

Background: Obsessive compulsive personality disorder (OCPD) is characterised by perfectionism, need for control and cognitive rigidity. Currently, little neuropsychological data exist on this condition, though emerging evidence does suggest that disorders marked by compulsivity, including OCD, are associated with impairment in cognitive flexibility and executive planning on neurocognitive tasks.

Aim: The current study investigated the neurocognitive profile in a non-clinical communitybased sample of people fulfilling diagnostic criteria for OCPD in the absence of major psychiatric comorbidity.

Method: Twenty-one non-clinical subjects who fulfilled DSM-IV criteria for OCPD were compared with 15 healthy controls on selected clinical and neurocognitive tasks. OCPD was measured using the Compulsive Personality Assessment Scale (CPAS). Participants completed tests from the Cambridge Automated Neuropsychological Test Battery including tests of set shifting (Intra-Extra Dimensional Set shifting: IED) executive planning (Stockings of Cambridge; SOC) and decision making (Cambridge Gamble Task; CGT).

Results: The OCPD group made significantly more IED-ED shift errors and total shift errors; and also showed longer mean initial thinking time on the SOC at moderate levels of difficulty. No differences emerged on the CGT.

Conclusions: Non-clinical cases of OCPD showed significant cognitive inflexibility coupled with executive planning deficits, whereas decision-making remained intact. This profile of impairment overlaps with that of OCD and implies common neuropsychological changes affect individuals with these disorders. 


\section{Introduction}

Obsessive-compulsive personality disorder (OCPD) is an early onset disorder characterised by perfectionism, need for control and cognitive rigidity ${ }^{1}$. It constitutes a relatively underresearched area of psychiatry and its nosological status is currently under review. Based on clinical similarities, arguments can be made for re-classifying OCPD together with obsessivecompulsive disorder $(\mathrm{OCD})^{2,3}$. To date, however, little clinical or neurosciences data address this issue, with few studies investigating 'pure' OCPD in the absence of other major psychiatric comorbidity. Therefore, OCPD was not included in the new DSM-5 obsessive compulsive and related disorder (OCRD) cluster, though its classification is undergoing review for the forthcoming World Health Organization ICD-11. A better understanding of the neuropsychological status of OCPD would help inform the debate.

The prevalence of obsessive-compulsive personality disorder is estimated to be high with up to almost $8 \%$ of the general population thought to be affected ${ }^{4}$. OCPD also represents one of the commonest personality disorders; a (weighted) life prevalence of $2 \%$ has been estimated in community samples ${ }^{5}$ with males and females roughly equally affected ${ }^{6}$. OCPD shares a high comorbidity with many psychiatric disorders, especially those characterised by compulsive behaviour, including OCD, body dysmorphic disorder and eating disorders ${ }^{7,8,2,9}$. Moreover, studies have demonstrated a higher prevalence of OCPD in cases of OCD compared with other 'non-compulsive' comparator disorders such as panic disorder, major depressive disorder ${ }^{10,11}$ and other forms of personality disorder ${ }^{12}$. Nevertheless, a high frequency of OCPD among individuals with OCD does not necessarily imply a unique, relationship between the two conditions ${ }^{13}$. Interestingly, several studies have also found a hereditary link between OCD and OCPD, suggesting that the first degree relatives of OCDaffected probands are more likely to suffer with OCPD than the first degree relatives of 
unaffected subjects; even after controlling for the potentially confounding effect of OCD in the relatives ${ }^{14}$ or OCPD in the probands ${ }^{15,16}$. These data provide stronger evidence for a psychopathological relationship between OCPD and OCD.

The overlap between OCD and OCPD may relate to similarities in symptom-profile that exist across the two disorders, such as perfectionism and preoccupation with detail and so the disorders may be confused ${ }^{17}$. Despite this, OCD can distinguished from OCPD by the absence of engagement in highly repetitive, distressing and disabling obsessions or compulsions $^{1,18}$. A recent study ${ }^{18}$ of OCPD patients revealed they were better able to delay gratification than OCD patients, and this was highlighted by the authors as key feature of the behavioural rigidity associated with OCPD. Notwithstanding such differences, emerging evidence suggests that disorders marked by compulsivity, including OCD and OCPD, are associated with shared aspects of neuropsychological impairment in specific domains including behavioural inhibition, cognitive flexibility and executive planning, that can be demonstrated using laboratory-based tasks ${ }^{19,20,21}$.

OCD patients have demonstrated deficits in pre-potent motor inhibition, measured on the stop-signal reaction time task ${ }^{21,22}$ (SSRT), attentional flexibility, measured on the intradimensional- extradimensional set-shift task (ID-ED), and executive planning on the Stockings of Cambridge (SOC) task ${ }^{23}$. A small controlled study suggested that patients with OCD and co-morbid OCPD demonstrated even greater cognitive inflexibility on the extradimensional set-shift paradigm than patients with OCD without co morbid OCPD ${ }^{3}$. A recent study ${ }^{24}$ investigating a small group of nonclinical participants with obsessive compulsive personality traits (OCPT) using similar tests, finding significant differences between OCPT and control participants on the Spatial Working Memory tasks, ID/ED tasks, Stockings of Cambridge, and the Dys-executive Questionnaire. These results suggest that a) a similar 
Original Research

range of executive dysfunction is present in people with prominent OCPT as in those with OCD, and b) a high convergence between clinical and ecological measures of executive functions in patients with compulsive disorders that may explain some of the clinical difficulties they experience, especially relating to their disabling behavioural rigidity and stubbornness.

The neuropsychological profile of OCD may extend beyond deficits in executive function to include emotional processing problems. Patients with OCD and trichotillomania have been compared on multiple aspects of cognitive function ${ }^{25}$; including affective processing using the affective no/go no test ${ }^{26}$. While patients with OCD and trichotillomania were no different at recognising happy words, the former made more errors in processing sad or negative emotion laden words. This finding is consistent with other recent studies suggesting that people with OCD are less able than healthy controls to recognise the facial expression of negative emotions ${ }^{27,28,29}$.

The aim of the current study was to investigate the neurocognitive profile of a non-clinical sample of people fulfilling diagnostic criteria for OCPD (in the absence of major psychiatric comorbidity). We particularly wished to discover the extent to which any deficits observed in OCPD resembled the published data for OCD. We chose to use a battery of neuropsychological tasks ${ }^{23}$, in order to be consistent with published OCD/OCPD/OCPT data $^{21,3,24}$. We selected a similar battery of tasks as used in the previous publications, including tests of emotion recognition and processing (affective go-no-go), executive planning (SOC) and attentional set shifting (ID-ED), as well as a decision-making task (Cambridge Gamble Task; CGT) that so far has not consistently been found to be impaired in 
OCD or OCPD (despite the evident decision-making difficulties observed in the psychopathology of these disorders).

\section{Methods}

OCPD participants were recruited via a screening advertisement that was e-mailed to 20,000 students at the University of Hertfordshire. The advert contained modified DSM-IV OCPD criteria, and students were asked to respond if they thought they had any of the traits. The advertisement was also placed on a website used by the students, advertised via a local newspaper and a local radio interview. The control participants were similarly recruited via email and word of mouth. Participants who responded were provided with participant information and given a minimum of 24 hours to consider participation. The protocol was approved by the local University research ethics committee and all participants provided written consent.

\section{Clinical assessment}

Each participant underwent a clinical assessment by psychiatrists with expertise in the assessment of Obsessive-Compulsive disorder (NAF \& GH). The assessment interview lasted 30 minutes and included administration of a brief demographic questionnaire and the Mini International Neuropsychiatric Inventory $(\mathrm{MINI})^{30}$, a structured screening interview designed to reliably identify most relevant DSM-IV mental disorders, supplemented by a semistructured clinical interview to identify other obsessive-compulsive related disorders not included in the MINI (such as body dysmorphic disorder, autistic spectrum disorder, Tourette's syndrome, hypochondriasis and trichotillomania). The diagnosis of OCPD was made using a semi-structured clinical interview involving questions directed at each of the 
DSM-IV 301.4 criteria, using the published criteria as the stem question for each item. In each case, all the diagnostic criteria were endorsed either positively or negatively. In addition, participants were questioned on the DSM-IV general diagnostic criteria for a personality disorder, to establish the extent to which the endorsed behaviours and traits impacted on the individual's life, to arrive at a decision as to whether a DSM-IV diagnosis was sustained. Syndrome-severity was additionally measured using the observer-rated Compulsive Personality Assessment Scale, $\left(\mathrm{CPAS}^{3}\right.$; See Figure one) which uses a semi-structured interview to provide a quantitative measure of the extent to which each of the eight DSM-IV or DSM-5 OCPD diagnostic behavioural/trait items is endorsed.

\section{Figure 1 about here}

\section{Participants}

Participants were aged between 18 and 65 years, and were required to have an adequate command of English. As noted above, participants recruited to the OCPD group were required to fulfil DSM-IV criteria for OCPD. Healthy controls were drawn from the same population and attempts were made, as far as possible, to match age, gender and IQ across the groups. For both groups, the presence of any other mental disorder, including depression, anxiety disorder, body dysmorphic disorder, OCD, obsessive compulsive related disorder, autistic spectrum disorder, anxiety disorder, depression, Tourette's syndrome, hypochondriasis, trichotillomania, resulted in exclusion. Participants in the control group were additionally required not to have any known history of mental illness and to score less than five out of a total of 32 on the CPAS, to ensure a low level of borderline OCPD traits. 
A total of 59 people applied to participate in the study and of those $31(52.5 \%)$ either withdrew or were not selected for participation. Of the 28 remaining, 4 were unsuitable after initial assessment owing to the presence of co-morbid disorders, 2 did not have high enough OCPD scores, and 1 person expressed reservations regarding participation. Thus the final OCPD group comprised 21 participants ( 9 females and 12 males; mean age $=26.5$; SD $=9.87$.

We assessed 21 healthy controls - three did not meet the inclusion/exclusion criteria and an additional two controls were later excluded because of extreme outlying data on cognitive tasks. Therefore, the control group comprised 15 participants (9 female and 6 males; mean age $=23.40 ; \mathrm{SD}=6.25)$.

\section{Neuropsychological Testing}

Following clinical assessment, participants completed the computerised neuropsychological test battery in a quiet room at the University of Hertfordshire. Participants were tested on the following tasks, including some derived from the Cambridge Neuropsychological Test Automated Battery $(\mathrm{CANTAB})^{23}$

\section{National Adult Reading Test (NART) $)^{31}$}

This is a measure of estimated 'premorbid intelligence', based on performance of reading aloud a list of irregular words. This assessment is scored according to the number of words pronounced correctly.

\section{Facial expression of emotion (FEEST) ${ }^{2}$}


This computerised task assesses the ability to recognise a range of facially-expressed emotions. The participants are presented with a series of standardised images of faces, each expressing an emotion, and required to select one of six emotion names presented as on the screen. The number of correct identifications is recorded. Patients with OCD and body dysmorphic disorder are known to perform poorly at recognising negative emotions such as anger or sadness on this $\operatorname{task}^{33,29}$.

\section{The Famous Face Test $(\text { FFT })^{33}$}

The famous face task investigates the processing and recognition of facial information. A set of 50 grey-scale photographs of famous people is inverted and presented to the participant at a set distance for a set time. The participants are asked to keep their head upright (resist the urge to tilt the head, which may offer an advantage in recognition of the face) while verbally identifying the famous faces. Scoring is he total number of faces correctly named. Patients with BDD are superior to healthy controls at recognising inverted faces ${ }^{33}$, suggesting the existence of disorder-related processing changes that may be related to a focus on processing topographical detail, as opposed to emotional expression ${ }^{33,34}$.

\section{Stockings of Cambridge Task (SOC)}

The SOC is a version of the Tower of London task $\mathrm{k}^{35}$, which assesses executive functioning and higher level cognition such as the ability to plan ahead. Participants are required to move a set of coloured balls shown on the bottom of the computer-screen, to match them up with another set of similar balls arranged at the top of the screen, as efficiently as possible i.e. with the minimum number of moves. The task is repeated with varying levels of difficulty. Performance on the task is measured by the thinking time required to make initial and all subsequent moves, the number of moves required to create the target arrangement and the number of tasks completed in the minimum number of moves. OCD patients have been 
shown to present with slowed planning and initial movements in the SOC task ${ }^{36}$ and therefore this experiment was used to assess whether OCPD shares aspects of the same neurocognitive impairment.

\section{Intra dimensional / Extra dimensional set-shift tasks (IDED)}

This test assesses rule acquisition and rule reversal ability, including the flexible adaptation of performance to suit the situation. Within the test, the participant is shown a pair of stimuli on the screen, composed of either a colour-filled object or a line drawing. At first, the participant performs the intra-dimensional task; for example, choosing between two different shapes of the colour-filled object based on trial and error and probabilistic computer feedback. After several trials, the rule governing the correct response is unpredictably changed to favour the alternative stimulus, i.e. the previously correct shape no longer produces positive endorsement by the computer. Through trial and error, the participant learns the new rule i.e. to switch responding to the alternate colour-filled shape. In the extradimensional switch (EDS) phase, an additional degree of complexity occurs whereby participants have to switch responding between different stimulus-dimensions e.g. between colour filled objects and line drawings. Performance on the task is measured by the number of trials required to meet a criterion level of response and the number of errors made. Similar to the SOC test, the ID-ED test is known to be affected in OCD, specifically at the most difficult EDS phase of the task.

\section{Cambridge Gambling Task (CGT)}

This task investigates decision-making and risk-taking behaviour, in the context of gambling for rewarding 'points'. Participants are required to select the location of a token hidden within a set of red or blue boxes on the screen. They are invited to gamble on how sure they are and can 'bet' points. The objective is to end up with the largest number of available 
points. CGT performance is judged using measures of deliberation time, quality of decision making, risk-taking and risk adjustment. The CGT task is not known to be impaired in OCD, though it is in impulse-control disorder such as gambling disorder and alcohol dependency ${ }^{37}$.

7. Six Elements Test (from The Behavioural Assessment of the Dysexecutive Syndrome Battery: $B A D S)^{38}$

The Six Elements test, a component of the BADS, investigates higher-order executive functions such as multi-tasking, planning and organizing. Participants are required to tackle three pairs of tasks (maths, picture naming and describing two events) within a 10 minute time limit; there are two versions of each task and the rules prohibit tackling these contiguously. Scoring is in terms of the number of tasks attempted, the number of times a rule is broken and the time spent on each activity. To the best of our knowledge, the this test has not been used to assess executive functions in OCD; however patients with schizophrenia and brain injury have shown deficits in this assessment ${ }^{39,40}$.

\section{Results}

The OCPD group scored significantly higher than controls on all aspects of the CPAS at a significant level $\mathrm{p}<.001$, apart from the Miserliness item ( $\mathrm{t}(34)=1.36$, OCPD 0.81 [SD 0.98] vs. controls 0.40 [SD 0.74]: $p=0.18$, Cohen's $d=0.48$ ), supporting the validity of the diagnostic grouping. The groups did not differ significantly in NART IQ (see Table one).

\section{Table one about here}

\section{FEEST and Famous Faces Tasks.}


A significant between-group difference emerged on the FEEST task, with a relative failure in the OCPD group to recognise one expression (surprise) out of a total of six different expressions; t (34) -2.67, OCPD 8.29 [SD 1.01] vs. controls 9.13 [0.83]: p=0.01, Cohen's d= -0.92. The Famous Face task also showed a numerical between-group difference with OCPD subjects naming fewer, although this marginally faileded to reach significance; $t$ (34) -1.93, OCPD 26.95 [SD 8.80] vs. controls 32.67 [SD-8.72]: $\mathrm{p}=0.06$, Cohen's $\mathrm{d}=-0.67$.

\section{Cognitive Flexibility - Intra Dimensional / Extra Dimensional Set-Shift}

The OCPD made more EDS errors than controls; $t$ (34) =2.33, OCPD 6.05 [SD 6.37] vs. controls 2.73 [SD 1.16]: $\mathrm{p}=0.03$, Cohen's $d=0.69$. Similarly, the OCPD group needed more trials to reach criterion on Stage 8; $\mathrm{t}(34)=2.27$, OCPD 6.81 [SD 8.10] vs. controls 2.73 [SD 1.16]: $\mathrm{p}=0.03$, Cohen's $\mathrm{d}=0.67$ and made more total errors (adjusted) items; $\mathrm{t}(34)=2.06$, OCPD 19.38 [SD 14.45] vs. controls 12.40 [SD 4.87]: $p=0.05$, Cohen's d= 0.62. No differences emerged for any pre-EDS stages (see Figure 2).

\section{Figure 2 about here}

\section{Stockings of Cambridge (SOC)}

The SOC task showed that the OCPD took longer than and controls for initial think time and this was significant at 3 moves; $\mathrm{t}(34)=2.03$, OCPD 5415.24 [SD 4367.44] vs. controls 3224.43 [SD-1955.81]: $\mathrm{p}=0.05$, Cohen's $\mathrm{d}=0.63$, and 4 moves; $\mathrm{t}$ (34) 3.35, OCPD 8925.17 [SD 6785.45] vs. control 3680.23 [SD 1984.51]: p<0.001, Cohen's d= 1.01; (See Figure 3).

There was also a non-significant trend toward a difference in mean subsequent thinking time on 4 moves; t (34) 1.80, OCPD 1124.58 [SD 2161.54] vs. controls 238.62 [SD 523.16]: $\mathrm{p}=.08$, Cohen's $\mathrm{d}=0.54$. 


\section{Cambridge Gambling Task (CGT)}

No significant between-group differences emerged on any outcome measure of the CGT.

\section{Six Elements Test (from the BADS $)^{38}$}

The Six Elements test revealed similar mean scores for both the OCPD and control groups; raw Score t $(34)=-.29$, OCPD 5.63 [SD 0.62] vs. controls 5.69 [SD 0.63]: p=.78, Cohen's $\mathrm{d}=-0.10$ and Profile Score t $(34)=-.48$, OCPD 3.69 [SD 0.63] vs. controls 3.77 [SD 0.44]: $\mathrm{p}=.64$, Cohen's $\mathrm{d}=-0.15$.

\section{Discussion}

Overall, these findings are consistent with the notion that OCPD shares a similar range of executive dysfunction with compulsive disorders such as OCD, in terms of cognitive inflexibility and impaired executive planning; and that b) the characteristics of the impairment might explain some of the associated clinical difficulties, such as behavioural rigidity, perfectionism and slowness.

As expected, clinical assessment with the Compulsive Personality Assessment Scale revealed significantly higher scores on: rigidity, preoccupation with details, perfectionism, workaholism, over-conscientiousness, hoarding and need for control, in our OCPD and control participants differing significantly on these items; reinforcing the strength of these specific characteristics as markers of OCPD ${ }^{17}$. Miserliness was the only OCPD trait that was significantly higher in the OCPD group. The DSM-IV and DSM-5 definition of OCPD includes two behavioural items not included in the ICD-10 definition, namely difficulty discarding (hoarding) and miserliness - both of which have been difficult to validate within 
the definition of $\mathrm{OCPD}^{41,42}$. Our results are consistent with the argument questioning the ongoing inclusion of miserliness as a reliable diagnostic criterion for OCPD.

Analysis of set-shifting ability on the ID-ED task showed that OCPD participants were significantly and substantially impaired on measures related specifically to the EDS. OCD patients are recognised to show robust deficits restricted to this domain within the $\operatorname{task}^{19}$. Moreover, a recent study found that the EDS deficit observed in a group with OCD comorbid with OCPD exceeded that of 'uncomplicated' OCD ${ }^{19}$, suggesting that overlapping pathophysiology existing in each disorder summates in the comorbid group. Aside from OCD and OCPD, the EDS deficit has been found in many mental disorders, including eating disorder ${ }^{43}$ body dysmorphic disorder ${ }^{44}$ and schizo-OCD ${ }^{45}$ - all disorders showing a high comorbidity with OCPD, though interestingly not so far seen in those with trichotillomania ${ }^{25}$. EDS impairment has been postulated as a neurocognitive marker of cognitive inflexibility and a key component of behavioural compulsivity ${ }^{19}$.

How then might this set-shifting deficit contribute toward the phenomenology of OCPD? Extreme perfectionism affecting task completion, excessive devotion to work, being overly inflexible, stubborn, rigid, judgmental and conscientious, can each be linked to a central deficiency in flexibly shifting thoughts and behaviour from one topic or activity to another, in response to changing environmental contingencies. Interestingly, patients with OCD may also use more rigid moral reasoning in response to impersonal moral dilemmas showing a thinking style characterised by reduced cognitive flexibility, demonstrating the convergence between clinical and ecological measures of cognitive inflexibility cross diagnostic groups controls $^{46}$.

Consistent with previous findings in the OCD literature ${ }^{21,47}$, participants with OCPD also differed from healthy controls on the SOC executive planning task, with deficits seen in the 
mean initial and subsequent thinking times that were restricted to the higher levels of task difficulty. This finding suggests that OCPD patients, along with OCD patients ${ }^{36}$, have a deficit in planning ahead and indicates that they think for much longer before attempting a complex task. Such deficits may well become manifest in OCPD phenomenology - indeed, interference in task completion represents a diagnostic criterion for OCPD. According to the DSM-5, such slowness is directly linked to the need to complete the task to a 'perfect' standard. A post-hoc analysis of our the data further investigated this relationship, revealing a significant correlation between Perfectionism scores and SOC mean initial thinking time at 3 moves $(\mathrm{r}(36)=0.44, \mathrm{p}=0.007)$. Thus, our results are consistent with such an account i.e. that the increased thinking time invested in executive planning represents the prioritisation of the perfect result over timeliness. Further research is, however, required to test whether executive slowing is determined by a perceived need to achieve perfection, or vice-versa. Other DSMIV and DSM-5 OCPD criteria, including difficulty discarding unused items, a reluctance to delegate tasks and miserliness, may also result from a weakness in planning capacity and too much time spent considering all the information before acting.

Like OCD, no significant impairment in decision-making or risk-taking was found on the CGT. No impairment emerged on the BADS tests. In contrast, the FEEST showed a single significant difference between OCPD and controls on the surprise item, which may simply represent a chance finding, but unlike OCD, no differences were found in recognising 'negative' emotions such as fear, anger and disgust $\mathrm{t}^{25,27,28,29}$. These results suggest OCPD may be associated with relatively less difficulty in emotional processing than OCD. In addition, the OCPD group did not show superior face recognition on the FFT, despite scoring highly on the CPAS item that measures attention to detail. This finding contradicted our expectation and suggests that the greater skill on the FFT seen in $\mathrm{BDD}^{33}$ may relate to other aspects of the disorder, such as a practice effect from habitually examining faces. 
Taken together, the results of this study are consistent with an overlapping cognitive inflexibility and executive planning impairment that is shared across OCPD and OCD, and in the case of cognitive inflexibility, shared also with some other compulsive disorders such as eating disorder ${ }^{48}$, body dysmorphic disorder ${ }^{44}$ and schizo-OCD ${ }^{45}$. These impairments may constitute a neurocognitive fingerprint for compulsivity and their clinical impact merits further clarification. Interestingly, OCPD subjects appeared relatively unimpaired on tasks of emotional processing, highlighting a potential area of difference with OCD and other obsessive compulsive and related disorders such as body dysmorphic disorder, where impaired recognition of negative expressions has been reported ${ }^{28}$. Importantly, in our OCPD sample, the neurocognitive dysfunction was observed in a non-clinical group of subjects who were not receiving any treatment whatsoever, and therefore cannot represent a confound of treatment. In the case of OCD, similar EDS deficits ${ }^{25}$, and possibly SOC changes $^{49}$, are also seen in unaffected first degree relatives and are thus thought to represent vulnerability markers, involving changes in structure and function within cortico-striatal neurocircuitry ${ }^{50}$. It would therefore be interesting to investigate the extent to which the relatives of subjects with OCPD demonstrate similar deficits.

\section{Limitations}

We aimed to identify, as far as possible, a relatively 'pure' form of non-comorbid OCPD. We employed an extensive screening test and interview and excluded any individuals who might criteria for major mental disorders including for example: major depressive disorder, OCD, body dysmorphic disorder, autistic spectrum disorder, Tourette's syndrome, hypochondriasis, and trichotillomania. Given that we were assessing a treatment-seeking group of individuals, we consider it unlikely that, for example, significant depressive symptoms would have affected our results. 
Recruiting participants in this category presented a challenge and therefore the number of participants was small. Future research should anticipate this difficulty, and provide enough time to recruit a larger number of participants.

The choice of neurocognitive task mainly focussed on domains known to be affected in OCRDs that are recognized as candidate trans-diagnostic markers of compulsive behaviour ${ }^{19}$. Deficits on these tasks also occur in disorders classified outside the OCRD grouping. Systematic mapping of a broader range of compulsive disorder using a comprehensive battery of neurocognitive tasks could help parse compulsivity across different disorders.

We have hypothesised mechanisms by which the deficits might influence the clinical phenomenology of OCPD and other OCRDs. However, the functional impact of these neurocognitive impairments still remains to be established with any degree of certainty. The development of trans-diagnostic rating scales for functional aspects of clinical compulsivity, such as the Cognitive Assessment Instrument of Obsessions and Compulsions (CAIOC-13), 13 would represent a relevant advance in this area ${ }^{51}$.

\section{Conclusions}

This study contributes toward the understanding of the neurocognitive basis of compulsivity across diagnoses. We have identified deficits in laboratory-based measures of cognitive flexibility and executive planning as significant findings in OCPD, which may potentially explain elements of the psychopathology and underpin disorder-related functional impairment. The neurocognitive profile for OCPD, in the absence of major psychiatric comorbidity, strongly resembled that seen for OCD. The results may be interpreted to 
support the classification of OCPD together with the OCRDs in the forthcoming WHO ICD11 classification. 


\section{References}

1 Butcher, J. N., Mineka, S., \& Hooley, J. M. Abnormal psychology. $14^{\text {th }}$ edition. Boston: Pearson Education INC; 2010

2 Murphy, D. L., Timpano, K. R., Wheaton, M. G., Miguel, E. C., \& Greenberg, B. D. Obsessivecompulsive disorder and its related disorders: a reappraisal of obsessive- compulsive spectrum concept. Dialogues in Clininical Neuroscience. 2010;12(2):131-148. Retrieved from http://www.ncbi.nlm.nih.gov/pmc/articles/PMC3181955/.

3 Fineberg, N. A., Sharma, P., Sivakumaran, T., Sahakian, B., \& Chamberlain, S. Does obsessive compulsive personality disorder belong within the obsessive-compulsive spectrum? CNS Spectrums. 2007;12(6):467-82. Retrieved from http://europepmc.org/abstract/MED/17545957.

4 Grant, B. F., Hasin, D. S., Stinson, F. S., Dawson, D. A., Chou, S. P., Ruan, W. J., \& Pickering, R. P. Prevalence, correlates, and disability of personality disorders in the United States: results from the national epidemiologic survey on alcohol and related conditions. Journal Clinical Psychiatry. 2004;65(7):948-958. Retrieved from http://www.ncbi.nlm.nih.gov/pubmed/15291684.

5 Torgersen S, Kringlen E, \& Cramer V. The prevalence of personality disorders in a community sample. Arch Gen Psychiatry. 2001;58:590-6.

6 Grant, J. E., Mooney, M. E., \& Kushner, M. G. Prevalence, correlates, and comorbidity of DSM-IV obsessive- compulsive personality disorder: results from the National Epidemiologic Survey on Alcohol and Related Conditions. J Psychiatr Res. 2012;46(4):469-75. doi:10.1016/j.jpsychires.2012.01.009.

7 Pinto, A., Mancebo, M. C., Eisen, J. L., Pagano. M. E., \& Rasmussen, S. A. The Brown Longitudinal Obsessive Compulsive Study: clinical features and symptoms of the sample at 
intake. Journal Clinical Psychiatry. 2006;67(5):703-11. Retrieved from http://www.ncbi.nlm.nih.gov/pmc/articles/PMC3272757/.

8 Coles, M.E., Pinto, A., Mancebo, M.C., Rasmussen, S.A., \& Eisen, J.L . (2008). OCD with comorbid OCPD: a subtype of OCD? Journal of Psychiatry Research. 2008;42:289-96. Retrieved from http://www.sciencedirect.com/science/article/pii/S0022395607000027.

9 Phillips, K.A., \& McElroy, S.L. Personality disorders and traits in patients with body dysmorphic disorder. Compr Psychiatry. 2000;41:229-36. Retrieved from http://www.sciencedirect.com/science/article/pii/S0010440X00980696\#.

10 Diaferia, G., Bianchi, I., Bianchi, M. L., Cavedini, P., Erzegovesi, S., \& Bellodi. Relationship between Obsessive-Compulsive Personality Disorder and Obsessive-Compulsive Disorder. Comprehensive Psychiatry. 1997,38;(1):38-42. Retrieved from http://www.sciencedirect.com/science/article/pii/S0010440X97900511.

11 Gordon, O. M., Salkovskis, P. M., Oldfeild, V. B., \& Carter, N. The association between obsessive compulsive disorder and obsessive compulsive personality disorder: Prevalence and clinical presentation. British journal of clinical psychology. 2013;52(3):300- 315. DOI: 10.1111/bjc.12016.

12 Albert, U., Maina, G., Forner, F., \& Bogetto, F. DSM-IV obsessive-compulsive personality disorder: Prevalence in patients with anxiety disorders and in healthy comparison subjects. Comprehensive Psychiatry. 2004;45(5):325-332. Retrieved from http://www.sciencedirect.com/science/article/pii/S0010440X04000690.

13 Starcevic, V., Berle, D., Brakoulias, V., Sammut, P., Moses, K., Milicevic, D. \& Hannan, A. Obsessive-compulsive personality disorder co-occurring with obsessive-compulsive disorder: Conceptual and clinical implications. Aust N Z J Psychiatry. 2013;47(1):65-73. doi:10.1177/0004867412450645. 
14 Calvo, R., Lazaro, L., Castro-Fornieles, J., Font, E., Moreno, E. \& Toro, J. Obsessive- compulsive personality disorder traits and personality dimensions in parents of children with obsessivecompulsive disorder. European Psychiatry.2009;24(3):201-6. doi: 10.1016/j.eurpsy.

15 Samuels, J., Nestadt, G., Bienvenu, O. J., Costa, P. T., Riddle, M. A., Liang, K. Y., Hoehn-Saric, R., Grados, M. A., \& Cullen, B. A. Personality disorders and normal personality dimensions in obsessive- compulsive disorder. Br J Psychiatry. 2000;177:457-62. doi:10.1192/bjp.177.5.457.

16 Bienvenu, O.J., Samuels, J.F., Wuyek, L.A., Liang, K.Y., Wang, Y., Grados, M.A. Is obsessivecompulsive disorder an anxiety disorder, and what, if any, are spectrum conditions? A family study perspective. Psychol Med. 2012;42(1):1-13. doi: 10.1017/S0033291711000742.

17 Eisen, J. L., Coles, M. E., Rasmussen, S. A. Clarifying the convergence between obsessive compulsive personality disorder criteria and obsessive compulsive disorder. Journal of Personality Disorders. 2006;20(3):294-305. Retrieved from http://www.ncbi.nlm.nih.gov/pmc/articles/PMC2548413/.

18 Pinto, A., Stienglass, J. E., Greene, A. L., Weber, E. U., \& Simpson, H. B. Capacity to delay reward differentiates obsessive- compulsive disorder and obsessive compulsive personality disorder. Biological Psychiatry. 2013;75(8):653-659. Retrieved from http://www.sciencedirect.com/science/article/pii/S0006322313008287.

19 Fineberg, N.A., Chamberlain, S.R., Goudriaan, A.E., Stein, D.J., Vanderschuren, L.J., Gillan, C.M., Shekar, S., Gorwood, P.A., Voon, V., Morein-Zamir, S., Denys, D., Sahakian, B.J., Moeller, F.G., Robbins, T.W., \& Potenza, M.N. New developments in human neurocognition: clinical, genetic, and brain imaging correlates of impulsivity and compulsivity. CNS Spectrums. 2014;19(1):69-89. doi:10.1017/S1092852913000801. 
20 Shin, M., Choi, H., Kim, H., Hwang, J., Kim, B., \& Cho, S. A study of neuropsychological deficit in children with obsessive-compulsive disorder. European Psychiatry. 2008;23(7):512-520. Retrieved from http://www.sciencedirect.com/science/article/pii/S0924933808015241.

21 Chamberlain, S. R., Fineberg, N. A., Blackwell, A. D., Robbins, T. W., \& Sahakian, B. J. Motor Inhibition and Cognitive Flexibility in Obsessive-Compulsive Disorder and Trichotillomania. The American Journal of Psychiatry. 2006;163:1282-1284. doi:10.1176/appi.ajp.163.7.1282.

22 Aron, A. R., Fletcher, P. C., Bullmore, E. T., Sahakian, B. J., \& Robbins, T. W. Stop-signal inhibition disrupted by damage to right inferior frontal gyrus in humans. Nature Neuroscience. 2003;6:115 - 116. doi:10.1038/nn1003.

23 Cambridge Cognition. (2014). http://www.camcog.com.

24 García-Villamisar, D. \& Dattilo, J. Executive Functioning in People with Obsessive-Compulsive Personality Traits: Evidence of Modest Impairment. Journal of Personality Disorders. 2013 (in press).doi: 10.1521/pedi_2013_27_101.

25 Chamberlain, S. R., Fineberg, N. A., Blackwell, A. D., Clark, L., Robbin, T. W., \& Sahakiana, B. J. A Neuropsychological Comparison of Obsessive- Compulsive Disorder and Trichotillomania. Neuropsychologia. 2007;45:654-662. Retrieved from http://www.sciencedirect.com/science/article/pii/S0028393206003150.

26 Murphy, F. C., Sahakian, B. J., Rubinsztein, J. S., Michael, A., Rogers, R. D., Robbins, T. W. Emotional bias and inhibitory control processes in mania and depression. [Abstract] Psychological Medicine. 1999;29(6):1307-1321. Retrieved from http://journals.cambridge.org/action/displayAbstract?fromPage=online\&aid=26163.

27 Aigner, M., Sachs, G., Bruckmuller, E., Winklbaur, B., Zitterl, W., Kryspin-Exner, I., Gur, R., \& Katschnig, H. Cognitive and emotion recognition deficits in obsessive-compulsive disorder. 
Psychiatry Research. 2007;149(1-3):121-128. Retrieved from

http://www.sciencedirect.com/science/article/pii/S0165178105004051.

28 Corcoran, K. M., Woody, S. R., \& Tolin, D. F. Recognition of facial expressions in obsessivecompulsive disorder. Journal of Anxiety Disorders. 2008;22(1):56-66. Retrieved from http://www.sciencedirect.com/science/article/pii/S0887618507000187.

29 Daros, A. R., Zakzanis, K. K., \& Recto, N. A. A quantitative analysis of facial emotion recognition in obsessive- compulsive disorder. Psychiatric Research. 2014;215(3):514-521. doi: 10.1016/j.psychres.2013.11.029.

30 Sheehan, D. V., Lecrubier, Y., Sheehan, K. H., Amorim, P., Janavs, J., Weiller, E., Hergueta, T., Baker, R., Dunbar, G. C. The Mini-International Neuropsychiatric Interview (M.I.N.I.): The Development and Validation of a Structured Diagnostic Psychiatric Interview for DSM-IV and ICD-10. Journal of Clinical Psychiatry. 1998;59(20):22-33. Retrieved from http://www.ncbi.nlm.nih.gov/pubmed/9881538.

31 Nelson, H. E., \& Willison, J. National Adult Reading Test (NART). Nfer-Nelson;1991.

32 Young, A.W., Perrett, D., Calder, A., Sprengelmeyer, R., Ekman, P. Facial expressions of emotion: stimuli and tests (FEEST). Suffolk (UK): Thurston, Thames Valley Test Company; 2002.

33 Jefferies, K., Laws, K. R., \& Fineberg, N. A. Superior face recognition in Body Dysmorphic Disorder. Journal of Obsessive-Compulsive and Related Disorders. 2012;1:175179.Retrieved from http://www.sciencedirect.com/science/article/pii/S2211364912000309.

34 Feusner, J. D., Moller, H., Altstein, K., Sugar, C., Bookheimer, S., Yoon, J., Hembacher, E. Inverted face processing in body dysmorphic disorder. Journal of Psychiatric Research. 2010;44(15):1088-94. doi: 10.1016/j.jpsychires.2010.03.015. 
35 Shallice, T. Specific Impairments in Planning. Philosophical Transactions of The Royal Society. 1982;298(1089):109-209. Retrieved from http://rstb.royalsocietypublishing.org/content/298/1089/199.short.

36 Nedeljkovic, M., Kyrios, M., Moulding, R., Doron, G., Wainwright, K., Pantelis, C., Purcell, R., \& Maruff, P. Differences in neuropsychological performance between subtypes of obsessivecompulsive disorder. Aust N Z J Psychiatry. 2009;43(3):216-26. doi: $10.1080 / 00048670802653273$

37 Lawrence, A. J., Luty, J., Bogdan, N. A., Sahakian, B. J., \& Clark, L. Problem gamblers share deficits in impulsive decision-making with alcohol-dependent individuals. Addiction. 2009;104:1006-1015. doi:10.1111/j.1360-0443.2009.02533.x

38 Wilson, B. A., Evans, J. J., Emslie, H., Alderman, N., \& Burgess, P. The Development of an Ecologically Valid Test for Assessing Patients with a Dysexecutive Syndrome. Neuropsychological Rehabilitation. 1998;8(3):213-228. doi: 10.1080/713755570.

39 Amann, B., Gomar, J. J., Ortiz-Gil, J., McKenna, P., Sans-Sansa, B., Sarro, S., Moro, N., Madre, M., Landin-Romeo, R., Vieta, E., Giokolea, J. M., Salvador, R., Pomarol-Clotet, E. Executive dysfunction and memory impairment in schizoaffective disorder: a comparison with bipolar disorder, schizophrenia and healthy controls. Psychological Medicine. 2012;42(10):21272135.

40 Evans, J. J., Chua, S. E., McKenna, P. J., \& Wilson, B. A. Assessment of the dysexecutive syndrome in schizophrenia. Psychological Medicine. 1997;27:635-646.

41 Ansell, E. B., Pinto, A., Edelen, M. O., \& Grilo, C. M. Structure of diagnostic and statistical manual of mental disorders, fourth edition criteria for obsessive-compulsive personality disorder in patients with binge eating disorder. Can J Psychiatry. 2008;53(12):863-7. 
42 Mataix-Cols, D., Frost, R. O., Pertusa, A., Clark, L. A., Saxena, S., Leckman, J. F., et al. Hoarding disorder: a new diagnosis for DSM-V? Depress Anxiety. 2010;27(6):556-572.

43 Roberts, M. E., Tchanturia, K., \& Treasure, J. L. Exploring the Neurocognitive Signatuve of Poor Set-Shifting in Anorexia and Bulimia Nervosa. Journal of Psychiatric Research. 2010;44(14):964-970. http://dx.doi.org/10.1016/j.jpsychires.2010.03.001.

44 Jefferies, K., Laws, K., Fineberg, N. A. Cognitive and perceptual processing in bdy dysmorphic disorder. European Neuropsychopharmacology. 2010;20(3). DOI:10.1016/S0924977X(10)70416-8.

45 Patel, D. D., Laws, K. R., Padhi, A., Farrow, J. M., Mukhopadhaya, K., Krishnaiah, R. \& Fineberg, N. A.The neuropsychology of the schizo-obsessive subtype of schizophrenia: a new analysis. Psychological Medicine. 2009;40:921-933.

46 Whitton, A. E., Henry, J. D., \& Grisham, J. R. Moral rigidity in obsessive-compulsive disorder: Do abnormalities in inhibitory control, cognitive flexibility and disgust play a role? Journal of Behaviour Therapy and Experimental Psychiatry. 2014;45(1):152-159. doi: 10.1016/j.jbtep.2013.10.001.

47 Kashyap, H., Kumar, J. K., Kandael, T., \& Reddy, Y. C. J. Neuropsychological functioning on Obsessive- Compulsive Disorder: Are Executive Functions the Key Deficit. Comprehensive Psychiatry. 2013;54(5):533-540. http://dx.doi.org/10.1016/j.comppsych.2012.12.003.

48 Tchanturia, K., Anderluh, M. B., Morris, R. G., Rabe-Heskerth, S., Collier, D. A., Sanchez, P., Treasure, J. L. Cognitive flexibility in anorexia nervosa and bulimia nervosa. Journal of the International Neuropsychological Society. 2004;10(4):513-520.

DOI: http://dx.doi.org/10.1017/S1355617704104086 
49 Vaghi, M.M.S., Hampshire A., Fineberg N.A., Bruhl A.B., Sahakian B.J., Chamberlain S.R., Robbins T.W. Neurocognitive endophenotype of Obsessive-Compulsive Disorder. Paper presented at: Organization for Human Brain mapping; 2014; Germany, Hamburg.

50 Fineberg NA, Robbins TW, Bullmore E, Potenza M, Menzies L, Chamberlain S, Sahakian B, Bechara A, Hollander E. Probing compulsive and impulsive behaviors, from animal models to endophenotypes: a narrative review. Neuropsychopharmacology 2010;35(3):591-604.

51 Dittrich, W. H., Johansen, T., \& Fineberg, N. A. Cognitive Assessment Instrument of Obsessions and Compulsions (CAIOC-13)- a new 13- item scale for evaluating functional impairment associated with OCD. Psychiatry Res. 2011;187(1-2):283-90. doi:

10.1016/j.psychres.2010.10.031. 
Table 1: Compulsive Personality Assessment Scale (CPAS) scores and background data for OCPD $(n=21)$ and Controls $(n=15)$

\begin{tabular}{|c|c|c|c|c|c|c|}
\hline & \multicolumn{2}{|l|}{ OCPD } & \multicolumn{2}{|c|}{ Control } & \multirow[t]{2}{*}{ p-value } & \multirow[t]{2}{*}{ Effect Size (d) } \\
\hline & Mean & $S D$ & Mean & $S D$ & & \\
\hline Age & 26.05 & 9.87 & 23.40 & 6.25 & 0.37 & 0.32 \\
\hline NART & 114.57 & 5.07 & 115.33 & 3.62 & 0.62 & -0.17 \\
\hline Preoccupation Details & 2.19 & 1.03 & 0.27 & 0.59 & $<0.001$ & 2.29 \\
\hline Perfectionism & 2.86 & 0.91 & 0.27 & 0.80 & $<0.001$ & 3.08 \\
\hline Workalholism & 2.24 & 1.41 & 0.40 & 0.63 & $<0.001$ & 1.64 \\
\hline Overconscientiousness & 2.19 & 0.87 & 0.27 & 0.46 & $<0.001$ & 2.71 \\
\hline Hoarding & 1.90 & 1.38 & 0.60 & 0.74 & $<0.001$ & 1.15 \\
\hline Control & 2.67 & 0.97 & 0.47 & 0.74 & $<0.001$ & 2.57 \\
\hline Miserliness & 0.81 & 0.98 & 0.40 & 0.74 & 0.18 & 0.48 \\
\hline Rigidity & 2.33 & 0.80 & 1.07 & 0.88 & $<0.001$ & 1.56 \\
\hline Total CPAS & 17.19 & 3.91 & 3.67 & 3.11 & $<0.001$ & 3.86 \\
\hline
\end{tabular}


Figure 1: Compulsive Personality Assessment Scale (CPAS)

\section{Compulsive Personality Assessment Scale (CPAS)}

Subject's name

Date of birth

Date of rating I

Rater's name

- _ _

Items refer to a stable pattern of enduring traits dating back to adolescence or early adulthood. Use the questions listed as part of a semi-structured interview.

For each item circle the appropriate score:

0 = absent 1 = mild $; 2$ = moderate; 3 = severe 4 = very severe .

\begin{tabular}{|l|l|l|l|l|l|}
\hline ITEM & \multicolumn{3}{|l|}{ RATING } \\
\hline $\begin{array}{l}\text { 1. Preoccupation with details } \\
\text { schedules to the extent that the major aim of the activity is lost? } \\
\text { scheocupied with details, rules, lists, order, organisation or }\end{array}$ & 0 & 1 & 2 & 3 & 4 \\
\hline $\begin{array}{l}\text { 2. Perfectionism } \\
\text { Would you describe yourself as a perfectionist who struggles with } \\
\text { completing the task at hand? }\end{array}$ & 0 & 1 & 2 & 3 & 4 \\
\hline $\begin{array}{l}\text { 3. Workaholism } \\
\text { Are you excessively devoted to work to the exclusion of leisure } \\
\text { activities and friendships? }\end{array}$ & 0 & 1 & 2 & 3 & 4 \\
\hline $\begin{array}{l}\text { 4. Over-conscientiousness } \\
\text { Would you describe yourself as over-conscientious and inflexible } \\
\text { about matters of morality, ethics or values? }\end{array}$ & 0 & 1 & 2 & 3 & 4 \\
\hline
\end{tabular}




\begin{tabular}{|c|c|c|c|c|c|}
\hline $\begin{array}{l}\text { 5. Hoarding } \\
\text { Are you unable to discard worn-out or worthless objects even when } \\
\text { they have no sentimental value? }\end{array}$ & 0 & 1 & 2 & 3 & 4 \\
\hline $\begin{array}{l}\text { 6. Need for control } \\
\text { Are you reluctant to delegate tasks or to work with others unless } \\
\text { they submit to exactly your way of doing things? }\end{array}$ & 0 & 1 & 2 & 3 & 4 \\
\hline $\begin{array}{l}\text { 7. Miserliness } \\
\text { Do you see money as something to be hoarded for future } \\
\text { catastrophes? }\end{array}$ & 0 & 1 & 2 & 3 & 4 \\
\hline $\begin{array}{l}\text { 8. Rigidity } \\
\text { Do you think you are rigid or stubborn? }\end{array}$ & 0 & 1 & 2 & 3 & 4 \\
\hline Tota & & & & & \\
\hline
\end{tabular}


Figure 2: Mean errors at each of the Intra Dimensional-Extra Dimensional stages (1-9);

Obsessive Compulsive Personality Disorder ( $n=21)$ vs. healthy controls $(n=15)$

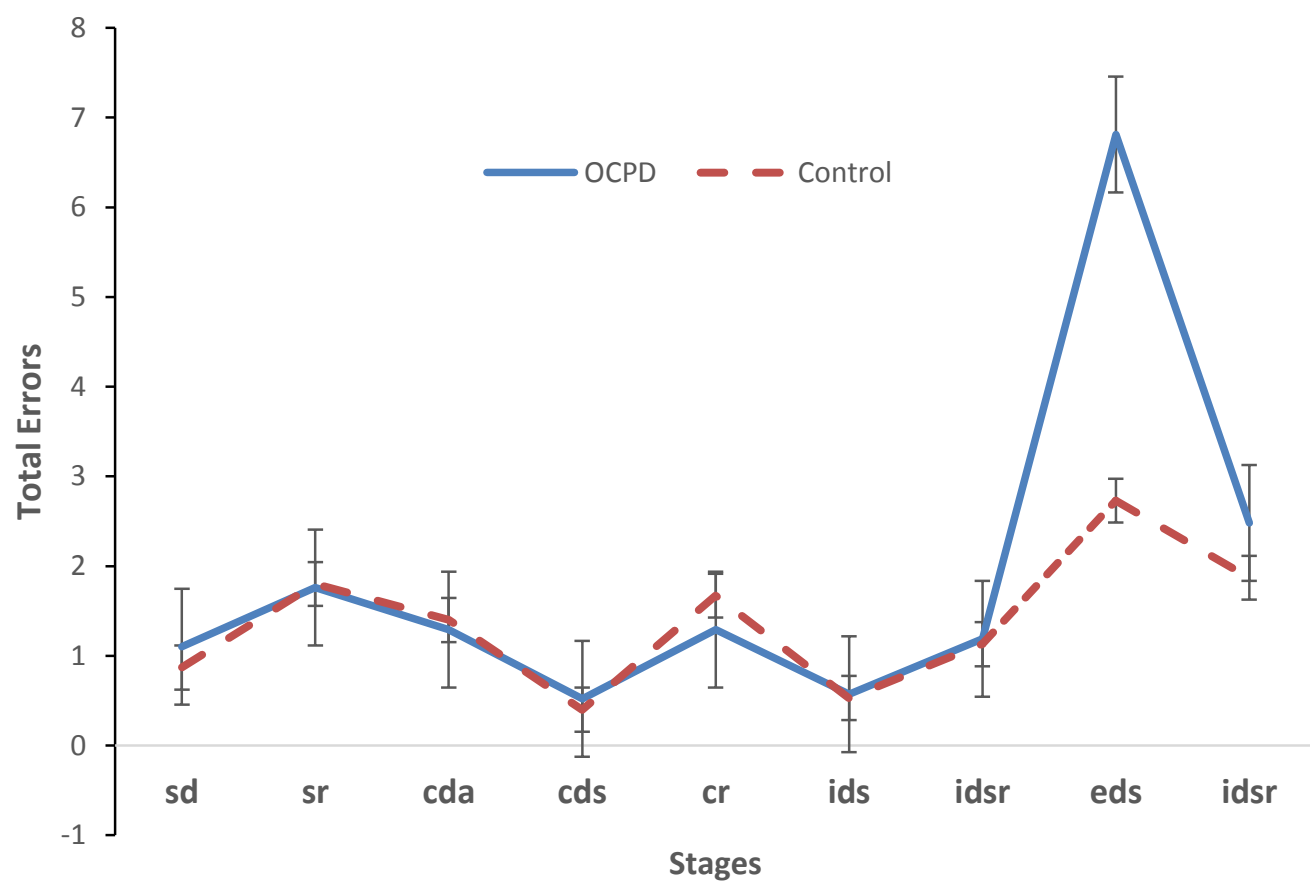

$(s d=$ simple discrimination; $s r=$ simple reversal; $c d a=$ compound discrimination adjacent; $c d s=$ compound discrimination superimposed; $c r=$ compound reversal; ids = intra-dimensional shift; idsr = intra-dimensional shift reversal; eds = extra-dimensional shift; edsr = extra-dimensional shift reversal).

(error bars $=\mathrm{SE}$ ) 
Original Research

Figure 3: Mean Initial Thinking Time (Msec) on the Stockings Of Cambridge task for OCPD (n=21) vs. healthy controls ( $n-15)$

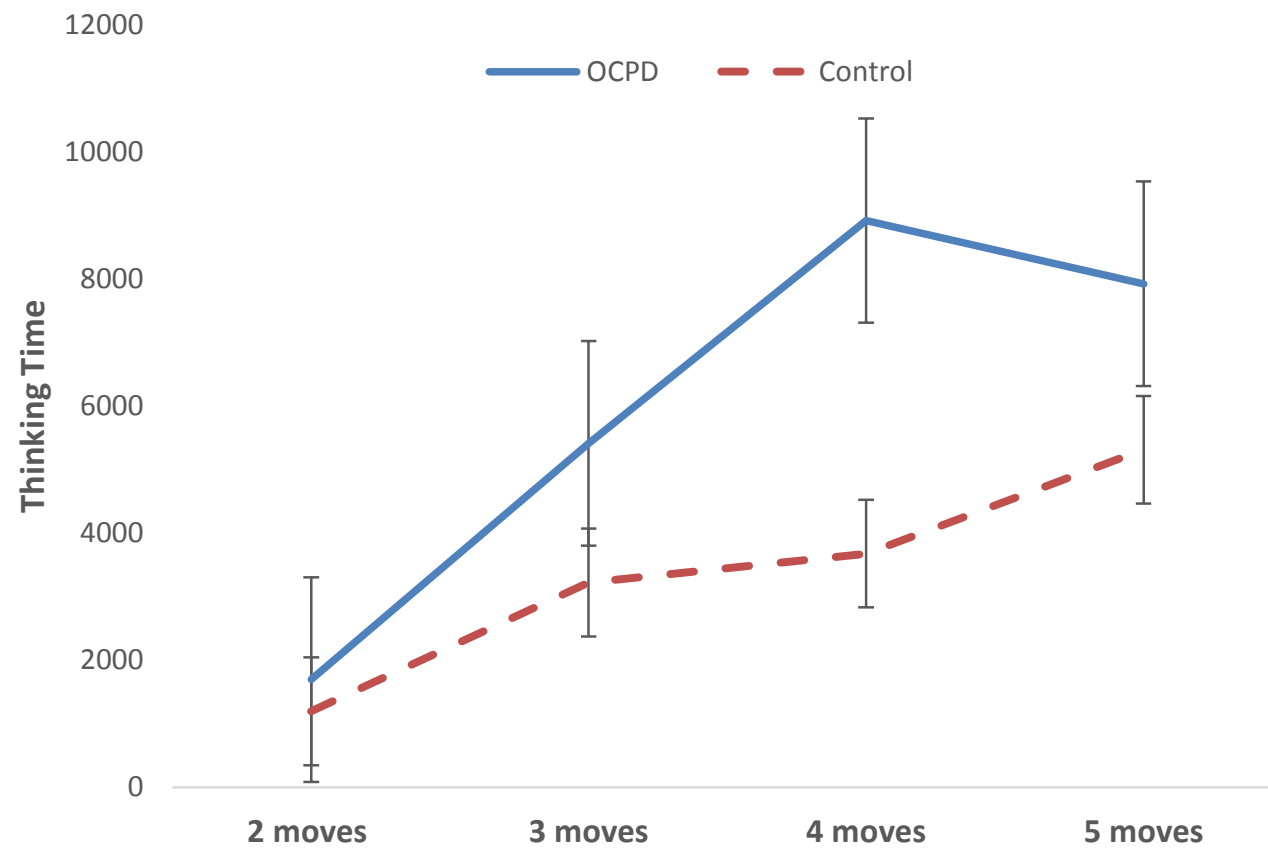

(error bars $=\mathrm{SE}$ ) 